\title{
Novel approaches to anesthesia in electroconvulsive therapy
}

\author{
Behzad Nazemroaya ${ }^{1 *}$ and Arash Pourreza ${ }^{2}$ \\ ${ }^{1}$ Department of Anesthesiology and Critical Care, School of Medicine, Isfahan University of Medical Sciences, Isfahan, Iran \\ ${ }^{2}$ Valiasr Hospital, Boroujen, Chaharmahal Bakhtiari, Iran
}

\begin{abstract}
Electroconvulsive therapy is one of the methods of treatment used in various kinds of psychiatric disorders including; depression, manic disorders, and catatonia. There are also different kinds of anesthetic approaches used in electroconvulsive therapy which include different kinds of anesthetic drugs, neuromuscular blocking agents and other medications. This article reviews the recent studies conducted regarding the various methods of anesthesia used in electroconvulsive therapy and proposes drugs and methods that could decrease unwanted side-effects and improve clinical outcomes of electroconvulsive therapy.
\end{abstract}

\section{Introduction}

Electroconvulsive therapy (ECT), also known as electroshock therapy is one of the methods of treatment in specific psychiatric diseases where minute electrical impulses are applied to the brain to treat many psychiatric disorders [1]. It is used in various kinds of psychiatric disorders, such as; depression, catatonia and manic disorders. Further indications are for those patients who have not shown any response to other drugs or those who are unable to do their daily activities because of these side effects or in patients with acute suicidal attempts. Furthermore, there are patients who have delusions or hallucinations or deep psychomotor retardation which may be less responsive to drugs, therefore early ECT may be indicated for them. There are a number of methods of applying ECT, one of which consists of 6 to 12 consecutive rounds of treatment over a timeframe of 2 to 4 weeks continued until showing significant clinical improvement. The seizures that are produced by ECT consist of a tonic phase which is 10 to 15 seconds long followed by a clonic phase which is 32 to 50 seconds long.

There are a few complications associated with the use of ECT. For instance, there may be some haemodynamic changes including severe bradycardia induced by vagal stimulation and mild hypertension afterwards. The stimulation of the sympathetic nervous system may cause hypertension and tachycardia lasting 5 to 10 minutes. There are also ECG variations and there may be changes like a prolongation in the interval in the pulse rate or an increase in the QT interval, in addition to inversion of the $t$ wave or arrhythmias such as atrial or ventricular arrhythmias.

Moreover, there may be elevated blood flow to the brain and an increase in the metabolic rate, which may cause a subsequent rise of the ICP. Cases of elevated intraocular or intragastric pressure have also been observed [2]. Regarding other side effects, the idea that ECT may cause brain damage has been speculated since the beginning of ECT treatments, but there are no data that would support this concept and on the contrary research has refuted it. For example, Devanand et al. [3] published a systematic review regarding this idea and found that there were no evidence that ECT may cause any brain damage or any damage at the structural or cellular level. Other serious medical outcomes with ECT are significantly rare.

There should be special consideration for patients with other coexisting medical diseases for example, cardiovascular diseases such as myocardial infarction of late, CHF or valvular heart disease. There may be other conditions which should be of concern, such as space occupying lesions or venous malformations, untreated diabetes, pulmonary diseases and osteoporosis. Evaluation before ECT is recommended, since it may identify coexisting medical diseases and the effect of ECT on such conditions [4].

Furthermore, there may be some cognitive side effects which may be of concern in ECT. Such cognitive side effects include disorders like, stereotypical and transient postictal disorientation which can be mild or severe in rare cases. Another type of cognitive disorders may be anterograde amnesia which is the inability to learn new information during and within a short time period after ECT treatment, which may have various degrees of severity. Another type of cognitive disorder is short-term retrograde amnesia which includes gaps in memory for occurrences a few weeks or even a few months prior to ECT. The fourth kind of cognitive disorder which is quite rare, maybe a more severe kind of retrograde memory loss where a patient suffers from persistent deficits in memory from several months or even years back.

There has not been enough controlled research conducted to show which patients may be at a higher risk for such negative effects or for providing pure explanation for these disorders.

${ }^{*}$ Correspondence to: Behzad Nazemroaya, Department of Anesthesiology and Critical Care, School of Medicine, Isfahan University of Medical Sciences, Isfahan, Iran, E-mail: behzad_nazem@med.mui.ac.ir

Key words: electroconvulsive therapy, preoxygenation, mask ventilation, muscle relaxants, nausea, vomiting

Received: January 20, 2019; Accepted: February 15, 2019; Published: February 20, 2019 
Additionally, there may be few adverse physical conditions after ECG which includes; headache, nausea, muscle pain, resulting from or subsequent to seizure or anesthesia or the combination of the two, but fortunately not clinically serious. Another side effect which may be observed in rare cases is known as "anaesthesia awareness". This may occur because of unpredicted differences in the response of patients to anaesthesia. In this condition the patient may not be able to move or breathe without being able to call the staff and alert them to the state of his or her consciousness. This condition may not be dangerous to the patient because there is sufficient oxygen but maybe very disturbing to the patient and require an adjustment to the analgesic requirements and a psychoeducational intervention. Therefore, it is paramount to inform patients to such side effects and to provide treatment for specific symptoms should they occur [4].

The contraindications of ECT include intracranial hypertension which is an absolute contraindication. Relative contraindications are the presence of a mass lesion inside the cranium with a normal ICP, aneurysm inside the cranium, myocardial infarction as of late, angina, congestive heart failure, severe glaucoma, bone fractures, thrombophlebitis, pregnancy and retinal detachment. Patients who are on constant treatment with benzodiazepines or lithium are recommended to discontinue these medications prior to ECT since, benzodiazepines may abolish or decrease seizure formation and lithium may cause post ECT delirium and confusion [4].

\section{Anesthesia for ECT}

Anesthesia is used in ECT to prevent unpleasant memories of the events while providing a prompt recovery to normal function. One of the complications of causing tonic- clonic seizures during ECT is the likelihood of creating injuries to the bones or fracture of vertebral bodies. In order to decrease such injuries neuromuscular blocking agents can be used to decrease the negative effects of such abrupt muscle contractions [1].

It is not recommended to provide premedication prior to the procedure, since, it may increase emergence time. In order to reduce secretions and to decrease bradycardia, anticholinergic medications are recommended. In patients with a history of nausea and vomiting ondansetron may be beneficial [2].

A small intravenous cannula can be placed for the administration of medications. Standard monitoring should be used. Preoxygenation is used for patients using $100 \%$ oxygen. Usually, the induction of anaesthesia is with methohexital, $1.5 \mathrm{mg}$ per $\mathrm{kg}$ IV or propofol $1 \mathrm{mg}$ per $\mathrm{kg}$ IV and succinylcholine, $1.0 \mathrm{mg}$ per $\mathrm{kg}$ IV. Afterwards, patients are ventilated using bag-mask ventilation with $100 \%$ oxygen. Small doses of nondepolarizing muscle relaxants can be used in patients who have contraindications for the use of succinylcholine. Using labetalol 10 to $50 \mathrm{mg}$ IV or Esmolol, 40 to $80 \mathrm{mg}$ IV prior to ECT may decrease the hypotensive effects of ECT and may be beneficial in those who suffer from hypertension or coronary artery disease. After ECT, Ketorolac can be used to decrease consequent pain [2].

In a recent study by Nazemroaya et al., in which preoxygenation with $100 \%$ oxygen was compared to bag-mask ventilation for ECT, it was shown that preoxygenation with $100 \%$ oxygen for 5 minutes, prevented the increase of heart rate and blood pressure without showing any significant decrease in $\mathrm{O}_{2}$ saturation. As a result, considering the high prevalence of tachycardia, elevated blood pressure and plummeting of oxygen saturation during ECT, preoxygenation using $100 \%$ oxygen prior to ECT could be an alternative to bag-mask ventilation during ECT which could improve clinical results after ECT and prevent vocational injuries to such as carpal tunnel syndrome to anesthesia technicians [5].

It may be prudent to pay extra attention to the concomitant use of other medications in patients with psychiatric diseases. Of importance are medications such as tricyclic antidepressants, monoamine oxidase inhibitors, selective serotonin reuptake inhibitors which require special care [2].

Various methods of anesthesia have been traditionally used and newer methods have also been utilised for anesthesia during ECT. In the traditional method, a number of agents are commonly used. As reviewed by Chanpatarana et al, the most common anesthetic agents include: methohexital, ketamine, thiopental, propofol and etomidate. Muscle relaxants such as succinylcholine and also nondepolarizing muscle relaxants are used to provide only moderate degrees of muscle relaxation and prevent injury to the musculoskeletal system, in addition to improving airway management. Atropine and glycopyrrolate are anticholinergic agents most commonly used to prevent bradycardia and asystole [6].

In addition, the use of antihypertensive medications may also be warranted in special circumstances. They include medications such as beta-blockers, nitroglycerine, trimethaphan, nicardipine and nitroprusside. Honarmand et al. [7] showed that using magnesiumsulfate reduces sympathetic responses caused by ECT but had no significant effect on seizure duration.

Ding et al. [8] also stated methohexital, thiopental, etomidate, propofol and ketamine as commonly used drugs during anesthesia for ECT.

Such medications can be used alone or in combination with each other, which have been used in ECT with different results, such as: propofol with ketamine, sodium thiopental with midazolam and propofol, methohexital and propofol or a mixture of sodium thiopental and propofol. Erdogan et al. [9] used a combination of ketamine and propofol and found that the seizure duration with this combination was similar to that of propofol, but the quality of seizures was superior in this combination compared to propofol alone. Also, the effects of propofol and ketamine in ECT in depressive patients were reviewed by Wang et al, who concluded that this combination may reduce the negative effects of ketamine alone and could be the first line of therapy in ECT in such patients. Nazemroaya et al. [10] reviewed the effects of ketamine compared to thiopental in ECT and found ketamine to be a suitable anesthetic agent in ECT despite some increase in heart rate, blood pressure and seizure duration. In another study by Nazemroaya et al. [11] which compared the cardiovascular effects of propofol to thiopental in ECT, they showed that the negative effects of propofol were more profound but verbal response time and recovery from anesthesia were quicker when using propofol. Shah et al. [12] compared thiopental, propofol and midazolam in ECT and expressed that propofol can be administered to ASA Grade 1 and 2 patients for a rapid and seamless induction and provide superior antiemetic, recovery with no interference with clinical outcomes while having the lowest increase in serum potassium levels. Erdil et al. [13] also concluded that propofol did not cause a prolongation of the QT interval and provided better hemodynamic stability compared to etomidate during ECT [14]. Furthermore, Nazemroaya et al. studied the effects of etomidate and thiopental during ECT and concluded that etomidate did not seem to show any negative interactions with the clinical outcome of ECT in patients. In addition, etomidate could also be used as a substitute 
in patients to prevent hemodynamic changes. The only complications were increased pain on injection and subsequent nausea and vomiting compared to thiopental [15]. In a study by Fredman et al. [16] propofol and methohexital were compared and it was found that propofol was a reliable anesthetic compared to methohexital during ECT, especially in elderly patients who needed ECT, since; it provided a more rapid recovery of cognitive function. In another study by Nazemroaya et al. [17] about the effects of midazolam in ECT, it was postulated that although midazolam could reduce seizure duration, but could also decrease headache, myalgia and nausea after ECT.

Various muscle relaxants have also been used in different researches including using succinylcholine and also nondepolarizing muscle relaxants. In research by Nazemroaya et al., hemodynamic changes after ECT were compared in succinylcholine and cisatracurium groups. They found that using low doses of cisatracurium caused shorter periods of recovery to spontaneous respiration after ECT and longer period of seizure during ECT, compared to succinylcholine. Unwanted complications that were observed were less in the cisatracurium group, making it a suitable alternative to succinylcholine in this procedure [18]. These findings were further confirmed by another study conducted by Nazemroaya et al. [19].

Further studies have also shown the efficacy of nondepolarizing neuromuscular blocking agents in ECT. Kadoi et al., studied the recovery period of rocuronium and compared it to succinylcholine. They subsequently observed that repeated doses of rocuroniumsugammadex did not have any negative complications including nausea, vomiting or QT interval prolongation. This combination also seemed to have safety and efficacy in patients who were otherwise susceptible to the adverse effects of succinylcholine [20].

\section{Conclusion}

ECT is an effective and prevalent method of treatment for many psychiatric patients. It is therefore important to study the various aspects of this mode of treatment especially anesthetic considerations for such a treatment. Many studies have been done to observe the negative effects of many anesthetic regimens and to prevent such adverse effects. These studies may have limitations, thus there need to be more studies conducted on a higher number of patients to improve anesthetic care during ECT and prevent adverse complications.

\section{References}

1. Mirzakhani H, Welch CA, Eikermann M, Nozari A (2012) Neuromuscular blocking agents for electroconvulsive therapy: a systematic review. Acta Anaesthesiol Scand 56: 3-16. [Crossref]

2. Pino RM (2016) Clinical Anesthesia Procedures of the Massachusetts General Hospital. 9th ed. Philadelphia, PA: Wolters Kluwer.

3. Devanand DP, Dwork AJ, Hutchinson ER, Bolwig TG, Sackeim HA (1994) Does ECT alter brain structure? Am J Psychiatry 151: 957-970. [Crossref]
4. Payne NA, Prudic J (2009) Electroconvulsive therapy: Part I. A perspective on the evolution and current practice of ECT. J Psychiatr Pract 15: 346-368. [Crossref]

5. Nazemroaya B, Shetabi H, Mohammadi S (2018) The Effects of Different Preoxygenation Techniques on Heart Rate and Blood Pressure Alterations in Patients Undergoing Electroconvulsive Therapy. J Isfahan Med Sch 36: 989-996.

6. Chanpattana W (2013) Anesthesia for ECT. German J Psychiatry 4: 33-39.

7. Honarmand A, Safavi SM, Salehi M, Habibzadeh M, Masoudifar M, et al. (2013) The Effect of Magnesium Sulfate on Blood Pressure and Heart Rate after Electroconvulsive Therapy. J Isfahan Med Sch 31: 1569-1578.

8. Ding Z, White PF (2002) Anesthesia for Electroconvulsive Therapy. Anesth Analg 94: 1351-1364. [Crossref]

9. Erdoğan Kayhan G, Yucel A, Colak YZ, Ozgul U, Yologlu SY, et al. (2012) Ketofol (mixture of ketamine and propofol) administration in electroconvulsive therapy Anaesth Intensive Care 40: 305-310. [Crossref]

10. Wang X, Chen Y, Zhou X, Liu F, Zhang T, et al. (2012) Effects of propofol and ketamine as combined anesthesia for electroconvulsive therapy in patients with depressive disorder. J ECT 28: 128-132. [Crossref]

11. Nazemroaya B, Shafa A, Khizab M (2016) Comparison of the Effect of Ketamine and Sodium Thiopental on Blood Pressure and Heart Rate during Electroconvulsive Therapy in Patients Admitted to the Ward of Psychiatry; A Double-Blind Randomized Clinical Trial. J Isfahan Med Sch 34: 1197-204. [Crossref]

12. Nazemroaya B, Sayedmoalemi S, Emami SA (2017) Comparison of Cardiovascular Effects of Propofol versus Sodium Thiopental Anesthesia in Children Undergoing Electroconvulsive Therapy (ECT). J Isfahan Med Sch 35: 463-469.

13. Shah PJ, Dubey KP, Watti C, Lalwani J (2010) Effectiveness of thiopentone, propofol and midazolam as an ideal intravenous anaesthetic agent for modified electroconvulsive therapy: A comparative study. Indian J Anaesth 54: 296-301. [Crossref]

14. Erdil F, Demirbilek S, Begec Z, Ozturk E, Ersoy MO (2009) Effects of propofol or etomidate on QT interval during electroconvulsive therapy. J ECT 25: 174-177. [Crossref]

15. Nazemroaya B, Babaei (2017) Comparison of Recovery Time and Complications During the Use of Etomidate and Thiopental Sodium in Anesthesia in Children for Electroconvulsive Therapy; A Double Blind Randomized Clinical Trial. Archives of Anesthesiology and Critical Care 3: 283-190.

16. Fredman B, Etienne J, Smith I, Husain MM, White PF (1994) Anesthesia fo electroconvulsive therapy: effects of propofol and methohexital on seizure activity and recovery. Anesth Analg 79: 75-79. [Crossref]

17. Nazemroaya B, Mohammadi AH, Najafian J, Moradi-Farsani D (2017) Effect of Preemptive Midazolam on Post-Electroconvulsive-Therapy (ECT) Headache, Myalgia, and Nausea and Vomiting. J Isfahan Med Sch 35: 26-31.

18. Nazemroaya B, Moradi-Farsani D, Sadeghi-Vaghfi A (2016) Comparison of Cisatracurium and Succinylcholine on Hemodynamic Changes during and after Electroconvulsive Therapy. J Isfahan Med Sch 34: 963-970.

19. Nazemroaya B, Hashemi ST, Pourreza A, Mirzakhani F (2018) Comparison of seizure duration and hemodynamic changes with cisatracurium versus succinylcholine in electroconvulsive therapy. Archives of Anesthesiology and Critical Care 4: 417-422.

20. Kadoi Y, Hoshi H, Nishida A, Saito S (2011) Comparison of recovery times from rocuronium-induced muscle relaxation after reversal with three different doses of sugammadex and succinylcholine during electroconvulsive therapy. $J$ Anesth 25: 855859. [Crossref]

Copyright: (C2019 Nazemroaya B. This is an open-access article distributed under the terms of the Creative Commons Attribution License, which permits unrestricted use, distribution, and reproduction in any medium, provided the original author and source are credited. 